\title{
Tilting complexes for group graded self-injective algebras
}

\author{
Andrei Marcus ${ }^{a}$ and Shengyong Pan ${ }^{b, *}$ \\ Faculty of Mathematics and Computer Science, \\ Babeş-Bolyai University, \\ Cluj-Napoca, Romania \\ E-mail: marcus@math.ubbcluj.ro \\ ${ }^{b}$ Department of Mathematics, \\ Beijing Jiaotong University, Beijing 100044, \\ People's Republic of China \\ E-mail: shypan@bjtu.edu.cn
}

\begin{abstract}
We construct derived equivalences between group graded self-injective algebras, starting from equivalences between their 1-components, obtained via a construction of J. Rickard and S. AlNofayee.
\end{abstract}

\section{Introduction}

Construction of tilting complexes for group graded algebras was primarily motivated by the problem of finding reduction methods for Broué's Abelian Defect Group Conjecture. In [6] two-sided tilting complexes are discussed, while in [7], Okuyamas's work promted the need for one-sided group graded tilting complexes. The paper [8] starts from a method, due to Rickard [11], to lift stable equivalences to derived equivalences by characterizing objects that correspond to simple modules. The context in [11] is that of symmetric algebras. This result has been generalized to self-injective algebras by Al-Nofayee [2], and then further extended by Rickard and Rouquier [12].

In this paper we obtain group graded derived equivalences between self-injective algebras starting from the main results of [2] and [12]. Thus we generalize here the main result of [8], and for this, we rely on the properties of the Nakayama functor in the group graded setting. In Section 2 we recall the definition and characterization of group graded tilting complexes, and we point out the it is no need to assume the finiteness of the group $G$. Our main results in Section 3 are group graded versions of [2, Theorem 4] and of [12, Theorem 3.9]. One of the applications in Section 4] is the combination of these results with Okuyama's strategy to lift stable equivalences. Another application is related to a construction of tilting complexes by Abe and Hoshino [1].

${ }^{*}$ Corresponding author. Email: shypan@ @jtu.edu.cn 2000 Mathematics Subject Classification: 18E30,16G10;16S10,18G15.

Keywords: self-injective algebra, graded derived equivalence, strongly graded algebra. 
In this paper, rings are associative with identity, and modules are left, unless otherwise specified. We denote by $A$-Mod the category of left $A$-modules, and by $A$-mod its full category consisting of finitely generated $A$-modules. If $X$ is an object of an additive category $\mathscr{A}, \operatorname{add}(X)$ denotes the full subcategory of $\mathscr{A}$ whose objects are direct summands of finite direct sums of copies of $X$. The notations $\mathscr{H}(\mathscr{A})$ and $\mathscr{D}(\mathscr{A})$ stand for the (unbounded) homotopy, respectively derived category of an abelian category $\mathscr{A}$. We freely use basic facts from [6], [7], [8].

\section{Preliminaries}

Let $k$ be a commutative ring and $G$ be a group (not necessarily finite). Suppose that $R=\bigoplus_{g \in G} R_{g}$ and $S=\bigoplus_{g \in G} S_{g}$ are $G$-graded $k$-algebras such that $R$ is $k$-flat. Throughout we denote by $A=R_{1}$ and $B=S_{1}$ the identity components of $R$ and $S$, respectively. We denote by $R$-Gr the category of $G$-graded $R$-modules, and by $R$-gr the category of finitely generated $G$-graded $R$-modules.

2.1. The group $G$ acts on $G$-graded $R$-modules $M \in R$-Gr by letting $M(g)=\bigoplus_{h \in G} M(g)_{h}$ be the $g$-suspension of $M$, where $M(g)_{h}=M_{h g}$ for all $g, h \in G$. If $R$ is strongly graded, then $G$ acts on $A$ modules $X \in A$-Mod by conjugation $X \mapsto R_{g} \otimes_{A} X$. Note that $\left(R \otimes_{A} X\right)(g)$ is naturally isomorphic to $R \otimes_{A}\left(R_{g} \otimes_{A} X\right)$ in $R$-Gr.

2.2. A $G$-graded $(R, S)$-bimodule $M$ can be regarded as an $R \otimes S^{\text {op }}$-module graded by the $G \times G$-set $G \times G / \delta(G)$, where $\delta(G)$ is the diagonal subgroup of $G \times G$, with 1-component $M_{1}$ a module over the diagonal subalgebra

$$
\Delta\left(R \otimes S^{\mathrm{op}}\right):=\left(R \otimes S^{\mathrm{op}}\right)_{\delta(G)}=\bigoplus_{g \in G} R_{g} \otimes S_{g^{-1}} .
$$

If $R$ and $S$ are strongly graded, then $M$ and $\left(R \otimes S^{\mathrm{op}}\right) \otimes_{\Delta(R \otimes S \text { op })} M_{1}$ are naturally isomorphic $G$-graded $(R, S)$-bimodules.

2.3. Recall that an object $\tilde{T}$ of $\mathscr{D}(R-\mathrm{Gr})$ is called a $G$-graded tilting complex if it satisfies the following conditions:

(i) $\tilde{T} \in R$-grperf; this means that, regarded as a complex of $R$-modules, $\tilde{T} \in R$-perf, that is $\tilde{T}$ is bounded, and its terms are finitely generated projective $R$-modules.

(ii) $\bigoplus_{g \in G} \operatorname{Hom}_{\mathscr{D}(R-\mathrm{Gr})}(\tilde{T}, \tilde{T}(g)[n])=0$ for $n \neq 0$.

(iii) $\operatorname{add}\{\tilde{T}(g) \mid g \in G\}$ generates $R$-grperf as a triangulated category.

The following result was proved in [7, Theorem 2.4] and [6, Theorem 4.7], based on Keller's approach [4], but note that the assumption that $G$ is finite is not needed.

Theorem 2.4. The following statements are equivalent:

(1) There is a G-graded tilting complex $\tilde{T} \in \mathscr{D}\left(R\right.$-Gr) and an isomorphism $S \simeq \operatorname{End}_{\mathscr{D}(R)}(\tilde{T})^{\text {op }}$ of G-graded algebras. 
(2) There is a complex $\tilde{U}$ of $G$-graded $(R, S)$-bimodules such that the functor

$$
\tilde{U} \otimes_{S}^{\mathbf{L}}-: \mathscr{D}(S) \longrightarrow \mathscr{D}(R)
$$

is an equivalence.

(3) There are equivalences

$$
F: \mathscr{D}(R) \longrightarrow \mathscr{D}(S) \quad \text { and } \quad F^{\mathrm{gr}}: \mathscr{D}(R-\mathrm{Gr}) \longrightarrow \mathscr{D}(S-\mathrm{Gr})
$$

of triangulated categories such that $F^{g r}$ is $G$-graded functor and the diagram

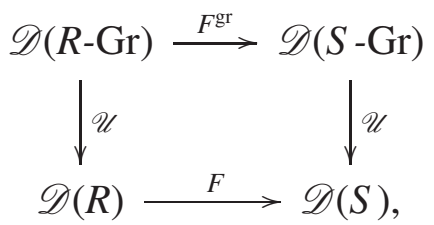

is commutative.

(4) There are equivalences

$$
F_{\text {perf }}: \mathscr{D}(R \text {-perf }) \longrightarrow \mathscr{D}(S \text {-perf }) \quad \text { and } \quad F_{\text {perf }}^{\mathrm{gr}}: \mathscr{D}(R \text {-grperf }) \longrightarrow \mathscr{D}(S \text {-grperf })
$$

of triangulated categories such that $F_{\text {perf }}^{\mathrm{gr}}$ is $G$-graded functor and $\mathscr{U} \circ F_{\text {perf }}^{\mathrm{gr}}=F_{\text {perf }} \circ \mathscr{U}$.

(5) (provided that $R$ and $S$ are strongly graded) There are (bounded) complexes $U$ of $\Delta\left(R \otimes S^{\text {op }}\right)$ modules and $V$ of $\Delta\left(S \otimes R^{\mathrm{op}}\right)$-modules, and isomorphisms $U \otimes_{B}^{\mathrm{L}} V \simeq A$ in $\mathscr{D}^{b}\left(\Delta\left(R \otimes R^{\mathrm{op}}\right)\right)$ and $V \otimes_{A}^{\mathrm{L}} U \simeq B$ in $\mathscr{D}^{b}\left(\Delta\left(S \otimes S^{\mathrm{op}}\right)\right)$.

Proof. We have an isomorphism $S \simeq \operatorname{End}_{\mathscr{D}(R)}(\tilde{T})^{\text {op }}$ of $G$-graded algebras for any group $G$, because $\tilde{T}$ is bounded, and each component of $\tilde{T}$ is finitely generated. It follows that the proofs of [7, Theorem 2.4] and [6, Theorem 4.7] carry over to the general situation.

2.5. A complex $\tilde{T} \in \mathscr{H}(R-\mathrm{Gr})$ is called $G$-invariant if $\tilde{T}(g) \simeq \tilde{T}$ in $\mathscr{H}(R$-Gr $)$ for all $g \in G$. More generally, $\tilde{T}$ is called weakly $G$-invariant if $\tilde{T}(g) \in \operatorname{add}(\tilde{T})$ in $\mathscr{H}(R$-Gr $)$ for all $g \in G$.

If $R$ is strongly $G$-graded, then a complex $T \in \mathscr{H}(A)$ is called $G$-invariant if $R_{g} \otimes_{A} T \simeq T$ in $\mathscr{H}(A)$ for all $g \in G$, and $T$ is called weakly $G$-invariant if $R_{g} \otimes_{A} T \in$ add $(T)$ in $\mathscr{H}(A)$ for all $g \in G$.

Note that if $R$ is strongly $G$-graded, then the functor $R \otimes_{A}-: A$-Mod $\rightarrow R$-Gr is an equivalence, hence a complex $T \in \mathscr{H}(R$-Gr) is $G$-invariant (weakly $G$-invariant) if and only if its identity component $T \in \mathscr{H}(A)$ is $G$-invariant (weakly $G$-invariant).

The following statement is also true for arbitrary $G$. It is essentially proved in [8, Proposition 2.1 and Remark 2.2], but for convenience, we include a proof here.

Proposition 2.6. Assume that $R$ is strongly G-graded. Let $\tilde{T}$ be a weakly G-invariant object in $\mathscr{K}^{b}(R-\mathrm{Gr})$. Denote by $T$ the identity component of $\tilde{T}$, and let $S=\operatorname{End} \mathscr{D}(R)(\tilde{T})^{\mathrm{op}}$. Then, $T$ is a tilting complex for $A$ if and only if $\tilde{T}$ is a G-graded tilting complex for $R$. Moreover, in this case, $S$ is strongly G-graded, and it is a crossed product if and only if $\tilde{T}$ is G-invariant. 
Proof. Since $R$ is strongly graded, the functor $R \otimes_{A}-: A$-Mod $\rightarrow R$-Gr is an equivalence, and a $G$ graded $R$-module is projective in $R$-Gr if and only if it is projective in $A$-Mod. It follows that $\tilde{T}$ is a bounded complex of finitely generated projective $R$-modules if and only if $T$ is a bounded complex of finitely generated projective $A$-modules. For each $m \in \mathbb{Z}$, we have

$$
\operatorname{Hom}_{\mathscr{K}(R)}(\tilde{T}, \tilde{T}[m]) \simeq \bigoplus_{g \in G} \operatorname{Hom} \mathscr{H}(R-\mathrm{Gr})(\tilde{T}, \tilde{T}[m](g))
$$

and

$$
\operatorname{Hom}_{\mathscr{H}(R-\mathrm{Gr})}(\tilde{T}, \tilde{T}[m](g)) \simeq \operatorname{Hom}_{\mathscr{H}(A)}\left(T, R_{g} \otimes T[m]\right)
$$

Since $\tilde{T}$ is weakly $G$-invariant, we have that for $m \neq 0$, $\operatorname{Hom}_{\mathscr{K}(A)}\left(T, R_{g} \otimes T[m]\right)=0$ if and only if $\operatorname{Hom}_{\mathscr{K}(R \text {-Gr })}(\tilde{T}, \tilde{T}[m](g))=0$. If $A$ is in the triangulated subcategory generated by add $(T)$ in $\mathscr{D}^{b}(A)$, then $R$ is in the triangulated subcategory generated by add $(\tilde{T})$ in $\mathscr{D}^{b}(R-\mathrm{Gr})$. Conversely, if $R$ belongs to the triangulated subcategory generated by add $(\tilde{T})$ in $\mathscr{D}^{b}(R-\mathrm{Gr})$, then ${ }_{A} R$ is in the triangulated subcategory generated by add $\left.{ }_{A} \tilde{T}\right)$ in $\mathscr{D}^{b}(A)$. Since ${ }_{A} R$ is a finite direct sum of copies of $A$, and ${ }_{A} \tilde{T}$ is a finite direct sum of copies of $T, A$ is in the triangulated subcategory generated by add $(T)$ in $\mathscr{D}^{b}(A)$. The last statement is clear, and also note that for the identity component of $S$ the have the isomorphism

$$
S_{1}=\operatorname{End}_{\mathscr{H}(R-\mathrm{Gr})}(\tilde{T}) \simeq \operatorname{End}_{\mathscr{H}(A)}(T)^{\mathrm{op}}
$$

of $k$-algebras.

\section{$3 \quad G$-graded self-injective algebras}

In this section we assume that $R$ is a strongly $G$-graded algebra over the field $k$, where $G$ is a finite group, and the identity component $A:=R_{1}$ is a finite dimensional algebra. For simplicity, we also assume that the field $k$ is algebraically closed, but the results below easily genelatize to arbitrary fields (see [11, Section 8]).

Proposition 3.1. Let $T$ be a $G$-invariant object in $\mathscr{H}(A)$, and denote $\tilde{T}=R \otimes_{A} T$ and $S=\operatorname{End}_{\mathscr{D}(R)}(\tilde{T})^{o p}$. If $T$ is a tilting complex for $A$ and $A$ is self-injective, then $S$ is a strongly G-graded self-injective algebra.

Proof. We know by Proposition 2.6 that $\tilde{T}$ is a $G$-graded tilting complex for $R$ and that $S$ is strongly $G$-graded. It is easy to see that $R$ is self-injective if and only if $A$ is self-injective (see, for instance, [6, 5.1]). Finally, self-injectivity is preserved by derived equivalences by [1, Lemmas 1.7 and 1.8] (see also [12, Corollary 3.12]).

Next we extend S. Al-Nofayee's construction [2] to the case of strongly $G$-graded algebras.

3.2. If there is a derived equivalence between the self-injective $k$-algebras $A$ and $B$, then the set $\mathcal{S}=$ $\left\{X_{i} \mid i \in I\right\}$ of objects corresponding to the simple $B$-modules, must satisfy the following conditions.

(a) $\operatorname{Hom}\left(X_{i}, X_{j}[m]\right)=0$ for $m<0$.

(b) $\operatorname{Hom}\left(X_{i}, X_{j}\right)=k$ if $i=j$ and 0 otherwise. 
(c) The objects $X_{i}, i \in I$, generate $\mathscr{D}^{b}(A$-mod) as a triangulated category.

(d) The Nakayama functor $v$ permutes the set $\mathcal{S}$, that is, there is a permutation $\sigma$ on $I$ such that $v\left(X_{i}\right)=X_{\sigma(i)}$.

In order to obtain a graded derived equivalence, we need to consider the conjugation action of $G$ on $A$-modules. Assume that $I$ is a finite $G$-set, and that the objects $X_{i}$ also satisfy the condition:

(e) $R_{g} \otimes_{A} X_{i} \simeq X_{g i}$ for all $g \in G$ and $i \in I$.

Lemma 3.3. Let $X_{i} \in \mathscr{D}^{b}(A$-mod), $i \in I$, be objects satisfying conditions 3.2 (a) to (e). There exist bounded complexes $T_{i}=I_{\mathcal{S}}\left(X_{i}\right)$ of finitely generated injective modules, and bounded complexes $T_{i}^{\prime}=$ $P_{\mathcal{S}}\left(X_{i}\right)$ of finitely generated projective modules, such that

$$
\begin{aligned}
& \operatorname{Hom}\left(T_{i}, X_{j}[m]\right)=\left\{\begin{array}{ll}
k, & \text { if } i=j \text { and } m=0 \\
0, & \text { otherwise. }
\end{array},\right. \\
& \operatorname{Hom}\left(X_{j}[m], T_{i}^{\prime}\right)=\left\{\begin{array}{ll}
k, & \text { if } i=j \text { and } m=0 \\
0, & \text { otherwise. }
\end{array},\right.
\end{aligned}
$$

and moreover,

$$
R_{g} \otimes_{A} T_{i} \simeq T_{g i}, \quad R_{g} \otimes_{A} T_{i}^{\prime} \simeq T_{g i}^{\prime},
$$

for all $g \in G$ and $i, j \in I$.

Proof. The proof given in [7, Theorem 2.4] is based on [11, Section 5], and it works for self-injective algebras. For convenience, we give a brief proof. Let $g \in G$ and $i \in I$. The construction of the complexes $T_{i}$ go by induction as follows.

Set $X_{i}^{(0)}:=X_{i}$, then $R_{g} \otimes_{A} X_{i}^{(0)}=X_{g i}^{(0)}$. By induction on $n$, we shall construct a sequence

$$
X_{i}^{(0)} \rightarrow X_{i}^{(1)} \rightarrow X_{i}^{(2)} \rightarrow \cdots \rightarrow X_{i}^{(n)} \rightarrow \cdots
$$

of objects and maps in $\mathscr{D}^{b}(A)$. Assuming that $X_{i}^{(n-1)}$ and $X_{g i}^{(n-1)}$ are constructed such that $R_{g} \otimes_{A} X_{i}^{(n-1)}=$ $X_{g i}^{(n-1)}$, we may construct that $X_{i}^{(n)}$ and $X_{g i}^{(n)}$ such that $R_{g} \otimes_{A} X_{i}^{(n)}=X_{g i}^{(n)}$ and we have the commutative diagram

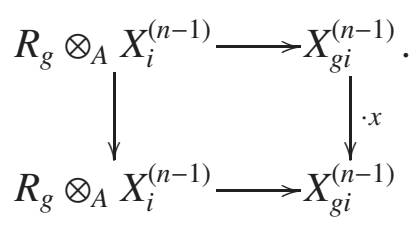

Finally, let $T_{i}=\operatorname{hocolim}\left(X_{i}^{(n)}\right)$, so it follows that $R_{g} \otimes_{A} T_{i} \simeq T_{g i}$.

Lemma 3.4. The permutation induced by the Nakayama functor $v$ commutes with the conjugation action of $G$ on A-modules. 
Proof. Recall that $v=D \operatorname{Hom}_{A}(-, A)$, where $D=\operatorname{Hom}_{k}(-, k)$. Since for any $g \in G$, the bimodule $R_{g}$ induces a Morita auto-equivalence of $A$-mod with quasi-inverse $R_{g^{-1}}$, for any $g \in G$ and $i \in I$ we have

$$
\begin{aligned}
v\left(R_{g} \otimes_{A} X_{i}\right) & \simeq \operatorname{DHom}_{A}\left(R_{g} \otimes_{A} X_{i}, A\right) \simeq D \operatorname{Hom}_{A}\left(X_{i}, \operatorname{Hom}_{A}\left(R_{g}, A\right)\right) \\
& \simeq D \operatorname{Hom}_{A}\left(X_{i}, R_{g^{-1}}\right) \simeq D\left(\operatorname{Hom}_{A}\left(X_{i}, A\right) \otimes_{A} R_{g^{-1}}\right) \\
& \simeq \operatorname{Hom}_{A}\left(R_{g^{-1}}, D \operatorname{Hom}_{A}\left(X_{i}, A\right)\right) \simeq \operatorname{Hom}_{A}\left(R_{g^{-1}}, v\left(X_{i}\right)\right) \\
& \left.\simeq \operatorname{Hom}_{A}\left(R_{g^{-1}}, A\right) \otimes v\left(X_{i}\right)\right) \simeq R_{g} \otimes_{A} v\left(X_{i}\right) \\
& \simeq X_{g \sigma(i)}
\end{aligned}
$$

On the other hand,

$$
v\left(R_{g} \otimes_{A} X_{i}\right) \simeq v\left(X_{g i}\right) \simeq X_{\sigma(g i)},
$$

so $\sigma(g i)=g \sigma(i)$ for all $g \in G$ and $i \in I$.

Theorem 3.5. Let $R$ be a strongly $G$-graded self-injective algebra with $R_{1}=A$, let I be a finite $G$-set, and let $X_{i} \in \mathscr{D}^{b}(A$-mod), $i \in I$, be objects satisfying conditions 3.2 (a) to (e).

Then there is another self-injective crossed product $G$-algebra $S$, and a $G$-graded derived equivalence between $R$ and $S$, whose restriction to $A$ sends $X_{i}, i \in I$, to the simple $S_{1}$-modules.

Proof. By [2, Lemma 5], there is a tilting complex $T=\bigoplus_{i \in I} T_{i}$ for $A$ such that

$$
\operatorname{Hom}\left(T_{i}, X_{j}[m]\right)= \begin{cases}k, & \text { if } \sigma(i)=j \text { and } m=0, \\ 0, & \text { otherwise. }\end{cases}
$$

It follows by Lemma 3.4 and by the definition of the homotopy colimit that $v\left(T_{i}\right) \simeq T_{\sigma(i)}$ for all $i \in I$ (see [2, Lemma 9]). By Lemma 3.3, the summands $T_{i}$ can be constructed to satisfy the additional condition

$$
R_{g} \otimes_{A} T_{i} \simeq T_{g i}
$$

for all $i \in I$ and $g \in G$. Consequently, $T$ is $G$-invariant, and Proposition 2.6 applies

3.6. Let $\mathcal{T}=\mathcal{D}^{b}(A)$, and let $\left(\mathcal{T}^{\leq 0}, \mathcal{T}^{>0}\right)$ be the bounded $t$-structure on $\mathcal{T}$ as in [12, Proposition 3.4]. Denote by $\mathcal{A}$ the heart of this $t$-structure, and by ${ }^{t} H^{0}$ the $H^{0}$-functor associated to this $t$-structure. Then the set of the simple objects of $\mathcal{A}$ is $\mathcal{S}$. Let $T_{i}=I_{\mathcal{S}}\left(X_{i}\right)$ and $T_{i}^{\prime}=P_{\mathcal{S}}\left(X_{i}\right), i \in I$, be the complexes defined in Lemma 3.3, and let $T^{\prime}=\bigoplus_{i \in I} T_{i}^{\prime}$.

Consider the finite dimensional $G$-graded DG algebra (see [7, 2.3])

$$
S=\operatorname{End}_{R}^{\bullet}\left(R \otimes_{A} \bigoplus_{i \in I} P_{\mathcal{S}}\left(X_{i}\right)\right)
$$

with 1-component

$$
B=\operatorname{End}_{A}^{\bullet}\left(\bigoplus_{i \in I} P_{\mathcal{S}}\left(X_{i}\right)\right)=\bigoplus_{m} \operatorname{Hom}_{A}\left(\bigoplus_{i \in I} P_{\mathcal{S}}\left(X_{i}\right), \bigoplus_{i \in I} P_{\mathcal{S}}\left(X_{i}\right)[m]\right) .
$$


We may now extend [12, Theorem 3.9] to strongly $G$-graded algebras.

Theorem 3.7. We have:

1) $H^{m}(S)=0$ for $m>0$ and for $m \ll 0$.

2) There is a $G$-graded derived equivalence $\mathscr{D}^{b}(S$-mod $) \simeq \mathscr{D}^{b}(R$-mod $)$.

3) There is a $G$-equivalence $H^{0}(B)-\bmod \simeq \mathcal{A}$.

Proof. 1) By [12, Theorem 3.9], we have that $H^{m}(B)=0$ for $m>0$ and for $m \ll 0$. Note that

$$
\operatorname{Hom}_{R}\left(R \otimes_{A} P_{\mathcal{S}}\left(X_{j}\right), R \otimes_{A} P_{\mathcal{S}}\left(X_{i}\right)[m]\right) \simeq \operatorname{Hom}_{A}\left(P_{\mathcal{S}}\left(X_{j}\right), \bigoplus_{g \in G} R_{g} \otimes_{A} P_{\mathcal{S}}\left(X_{i}\right)[m]\right),
$$

and, by Lemma 3.3, $\operatorname{Hom}\left(P_{\mathcal{S}}\left(X_{j}\right), R_{g} \otimes_{A} P_{\mathcal{S}}\left(X_{i}\right)[m]\right)=0$ for all $i, j \in I$ if and only if $\operatorname{Hom}\left(P_{\mathcal{S}}\left(X_{j}\right), P_{\mathcal{S}}\left(X_{i}\right)[m]\right)=0$ for all $i, j \in I$. Consequently, $H^{i}(S)=0$ for $m>0$ and for $m \ll 0$.

2) We also know that the functor

$$
\operatorname{Hom}_{A}\left(\bigoplus_{i \in I} P_{\mathcal{S}}\left(X_{i}\right),-\right): \mathscr{D}^{b}(A) \rightarrow \mathscr{D}^{b}(B)
$$

is an equivalence. Since $R \otimes_{A}-: A$-Mod $\rightarrow R$-Gr is an equivalence, and a $G$-graded $R$-module is projective in $R$-Gr if and only if it is projective in $A$-Mod, it is clear that $R_{A} \otimes_{A}\left(\bigoplus_{i \in I} P_{\mathcal{S}}\left(X_{i}\right)\right)$ is perfect object in $\mathscr{D}(R-\mathrm{Gr})$ if and only if $\bigoplus_{i \in I} P_{\mathcal{S}}\left(X_{i}\right)$ is a perfect complex of $A$-modules. Therefore, we get the $G$-graded derived equivalence

$$
\operatorname{Hom}_{R}^{\bullet}\left(R \otimes_{A} \bigoplus_{i \in I} P_{\mathcal{S}}\left(X_{i}\right),-\right): \mathscr{D}^{b}(R \text {-mod }) \rightarrow \mathscr{D}^{b}(S \text {-mod })
$$

3) By [12, Theorem 3.9], ${ }^{t} H^{0}\left(T^{\prime}\right)$ is a progenerator for $\mathcal{A}$ with endomorphism algebra $H^{0}(B)$. As in Lemma 3.3, $T^{\prime}$ is $G$-invariant, hence ${ }^{t} H^{0}\left(T^{\prime}\right)$ is also $G$-invariant, and the statement follows.

\section{Applications and examples}

Okuyama's strategy to lift a stable equivalence to a derived equivalence also generalizes to strongly $G$-graded self-injective algebras. We assume that $k$ is a field, and here we need to assume in addition that the order of $G$ is invertible in $k$, that is, the characteristic of $k$ does not divide $|G|$.

Corollary 4.1. Let $R$ and $S$ be strongly $G$-graded self-injective algebras. Assume that $|G|$ is invertible in $k$, and let $M$ be a $G$-graded $R$-S-bimodule inducing a stable equivalence of Morita type between $R$ and $S$.

If there are objects $X_{i} \in \mathscr{D}^{b}(A$-mod), $i \in I$, be objects satisfying conditions 3.2 (a) to (e), and such that $X_{i}$ is stably isomorphic to $M_{1} \otimes_{B} S_{i}$, for all $i \in I$, then there is a G-graded derived equivalence between $R$ and $S$.

Proof. By Theorem 3.5 there is a self-injective crossed product $R^{\prime}$ and a $G$-graded derived equivalence between $R$ and $R^{\prime}$ By [7, Remark 3.4], we obtain a $G$-graded stable equivalence of Morita type between $R$ and $R^{\prime}$. Consequently, we have a stable Morita equivalence between $R^{\prime}$ and $S$ induced by a $G$ graded $R^{\prime} \otimes S^{\text {op }}$-bimodule $M^{\prime}$. Since simple $A^{\prime}$-modules are sent to simple $B$-modules, a theorem 
of Linckelmann [5, Theorem 2.1] says that a direct $A \otimes B^{\mathrm{op}}$-summand $M$ of $M_{1}^{\prime}$ induces a Morita equivalence between $A^{\prime}$ and $B$. Since $|G|$ is invertible in $k$, we have that $M$ is a $\Delta\left(R^{\prime} \otimes S^{\text {op }}\right)$-summand of $M_{1}^{\prime}$, so by [6, Theorem 3.4], $\left(R^{\prime} \otimes S^{\text {op }}\right) \otimes_{\Delta\left(R^{\prime} \otimes S^{\text {op }}\right)} M$ induces a $G$-graded Morita equivalence between $R^{\prime}$ and $S$. By composing this equivalence with the $G$-graded derived equivalence between $R$ and $R^{\prime}$, we obtain a $G$-graded derived equivalence between $R$ and $S$.

Remark 4.2. By [2, Section 4] and [11, Section 6.3], we must have

$$
X_{i} \simeq \Omega^{n_{i}}\left(M_{1} \otimes_{B} S_{i}\right)\left[n_{i}\right] .
$$

Here we only have to verify condition 3.2 (e). But this follows immediately since $R_{g} \otimes_{A} M \otimes_{B} S_{g^{-1}} \simeq M$ as $(A, B)$-bimodules, and the syzygy functor $\Omega$ also commutes with the $G$-conjugation functor $R_{g} \otimes_{A}-$.

Another construction of tilting complexes for self-injective algebras is given in [1, Section 3] in the case of representation-finite algebras. Here we adapt [1, Theorem 3.6] in order to obtain a $G$-invariant tilting complex, so that Proposition 2.6 can be applied. Here $G$ is not assumed to be finite.

Proposition 4.3. Assume that $A$ is representation-finite. Let $P$ be a bounded complex of finitely generated projective A-modules such that $\operatorname{Hom}_{\mathscr{H}(A)}\left(P, R_{g} \otimes_{A} P[m]\right)=0$ for all $m \neq 0$ and $g \in G$, and add $P=\operatorname{add} v P$. Then there exists a bounded complex of finitely generated projective A-modules $Q$ such that $Q \oplus P$ is a $G$-invariant tilting complex.

Proof. By adding $G$-conjugates of $P$, we may assume that $P$ is $G$-invariant. The complex $P$ defines a torsion theory $(\mathscr{T}, \mathscr{F})$, where $\mathscr{T}={ }^{\perp} H^{0}(P)$ and $\mathscr{F}=\mathscr{T}^{\perp}$ are invariant under the Nakayama functor $v$. Since $P$ is $G$-invariant, we have that $\mathscr{T}$ and $\mathscr{F}$ are also closed under $G$-conjugation. Let $\left\{e_{j} \mid j \in J\right\}$ be a basic set of orthogonal local idempotents in $A$. The $G$-conjugation action of $G$ on $A$-modules induces a $G$-set structure on $J$, such that then the subsets $J_{1}=\left\{j \in J \mid A e_{j} \in \mathscr{T}\right\}$ and $J_{2}=\left\{j \in J \mid A e_{j} \in \mathscr{F}\right\}$ are $G$-stable. On can easily deduce from these observations and 3.4 that the tilting complex $T$ constructed in [1, Lemma 3.4] and the complex $Q$ from the proof of [1, Theorem 3.6] are $G$-invariant.

Example 4.4. This example is related to [3, Example 9.5]. Let $A$ be a finite dimensional $k$-algebra given by the quiver

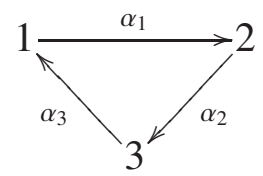

with relations

$$
\alpha_{1} \alpha_{2} \alpha_{3} \alpha_{1}=\alpha_{2} \alpha_{3} \alpha_{1} \alpha_{2}=\alpha_{3} \alpha_{1} \alpha_{2} \alpha_{3}=0 .
$$

Let $P=P_{2} \oplus P_{3}$. Then add $P=\operatorname{add} v(P)$. Then there is a complex

$$
Q:=0 \rightarrow P_{2} \rightarrow P_{1} \rightarrow 0
$$

with $P_{2}$ in degree 0 such that $P \oplus Q$ is a tilting complex for $A$, and the endomorphism algebra $B$ of $P \oplus Q$ is the algebra given by the quiver

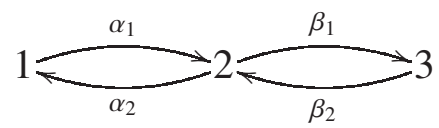


with relations

$$
\alpha_{1} \beta_{1}=\beta_{2} \alpha_{2}=0, \alpha_{1} \alpha_{2} \alpha_{1}=\beta_{2} \beta_{1} \beta_{2}=\alpha_{2} \alpha_{1}-\beta_{1} \beta_{2}=0
$$

Moreover, consider the infinite cyclic group $G=\left\langle g, g^{-1} \mid g g^{-1}=g^{-1} g=1\right\rangle$ acting on $A$ and $B$ as follows: The element $g$ fixes all the vertices and the edge $\alpha_{1}$ in $A$, and $g\left(\alpha_{i}\right)=\alpha_{i}+\alpha_{i} \alpha_{i+1} \alpha_{i+2} \alpha_{i}$ $(\bmod 3)$ for $i \neq 1$, while $g$ fixes all the vertices and all $\alpha_{i}$ in $B$, and $g\left(\beta_{i}\right)=\beta_{i}+\beta_{i} \beta_{i+1} \beta_{i}(\bmod 2)$ for $i \neq 1$. Then the complexes $P$ and $Q$ are $G$-invariant, and $R \otimes_{A}(P \oplus Q)$ induces a $G$-graded derived equivalence between the skew group algebras $R=A * G$ and $S=B * G$.

Example 4.5. Let $A$ be a finite dimensional $k$-algebra given by the quiver

$$
1 \stackrel{\alpha_{1}}{\longrightarrow} 3 \stackrel{\alpha_{2}}{\longleftarrow} 2
$$

(see [10, 1.4] and [9, Example (2)]). Let $G=\{1, g\}$, with $g$ acting on $A$ by interchanging vertices 1 and 2 , and consider the skew group algebra $R=A * G$. Let $T=P_{1} \oplus P_{2} \oplus I_{3}$. Then $R \otimes_{A} T$ is a $G$-graded tilting complex for $R$.

Acknowledgements. Shengyong Pan is funded by China Scholarship Council.

The authors thank the referee for his/her observations which improved the presentation of the paper.

\section{References}

[1] H. Aвe and M. Hoshino, On derived equivalences for selfinjective algebras, Comm. Algebra 34 (2006), $4441-4452$.

[2] S. Al-NofaYEe, Equivalences of derived categories for self-injective algebras, J. Algebra 313 (2007), 897904.

[3] H. Asashiba, A generalization of Gabriel's Galois covering functors and derived equivalences, J. Algebra 334 (2011), 109-149.

[4] B. KelLeR, On the construction of triangle equivalences. In: Derived equivalences for group rings (SpringerVerlag, 1998), pp. 155-176.

[5] M. Linckelmann, Stable equivalences of Morita type for self-injective algebras and p-groups, Math. Z. 223 (1996) 87-100.

[6] A. Marcus, Equivalences induced by graded bimodules, Comm. Algebra 26 (1998), 713-731.

[7] A. Marcus, Tilting complexes for group graded algebras, Journal of Group Theory 6 (2003), 175-193.

[8] A. Marcus, Tilting complexes for group graded algebras II, Osaka J. Math. 42 (2005), no.2, 453-462.

[9] J. I. Mryachi, Extensions of rings and tilting complexes, J. Pure Appl. Algebra 105 (1995), 183-194.

[10] I. Reiten and C. Riedtmann, Skew group algebras in the representation theory of Artin algebras, J. Algebra 92 (1985), 224-282.

[11] J. Rickard, Equivalences of derived categories for symmetric algebras, J. Algebra 257 (2002), 460-481.

[12] J. Rickard and R. RouQuier, Stable categories and reconstruction, J. Algebra 475 (2017), 287-307. 